INTERNATIONAL JOURNAL OF SYSTEMATIC BACTERIOLOGY

Vol. 20, No. 4 October 1970

pp. $475-482$

Copyright 1970, Iowa State University Press

\title{
CELL WALL COMPOSITION IN THE CLASSIFICATION OF STREPTOCOCCI
}

\author{
Harry Gooder \\ Department of Bacteriology and Immunology \\ School of Medicine, University of North.Carolina \\ Chapel Hill
}

As a genus streptococci are usually immediately recognizable, due to their characteristic appearance. However, classification at the subgroup or species level is unsatisfactory. Frequently the possession of a single character, e.g., the serological group antigen or bile solubility, has been used to assign organisms to a species such as Streptococcus pyogenes or Diplococcus pneumoniae. Recent attempts to obtain a satisfactory classification have utilized methods ranging from the molecular structure of deoxyribonucleic acid (DNA) to computer analysis. Weissmann et al. (1966), using the DNA/RNA homology technique, suggested a clos $\bar{e}$ genetic relationship between streptococci of Lancefield Groups $A$ and $C$ and a remote genetic relationship between these streptococci and S. faecalis. This method was sufficiently sensitive to differentiate between the two serotypes of the same group. In theory this DNA-RNA hybridization technique and other similar approaches are assessing the potential genetic relatedness between two organisms at the molecular level. For a recent review see Mandel (1969). In contrast, the numerical taxonomy approach is to assess relatedness from the overall similarity of the phenotypic expression of a very large number of characters as revealed by the "clustering" of organisms with shared combinations of characteristics. As utilized by Colman (1968), Raj and Colwell (1966) and Drucker and Melville (1969), this technique has revealed clusters of strains with attributes distributed in a nonrandom manner. However, the clusters are demonstrated by some computer programs and not by others and furthermore often show little relationship to the classification schemes currently accepted, for example, for the $\beta$-hemolytic streptococci. Neither the genetic nor numerical taxonomic approach has yet gained wide acceptance as a practical method for the routine classification of streptococci.

Any general textbook of bacteriology lists a large, some would say voluminous, number of streptococcal species arranged in a classification based on convenient laboratory tests that have been developed for the identification of an isolated but unknown streptococcus. It is useful initially to remind ourselves of the major features of this classification scheme. Neglecting the obvious required features of the group, for example, that it be a Gram-positive coccus, the techniques found useful can be classified into a small number of categories. These are:

a) The source of the strain. This invariably colors our approach to its identification.

b) Physiological tests which depend on an activity of the organism, e.g., reaction on blood agar, growth temperature, salt tolerance, 
bile tolerance, ammonia production from arginine; and similar methods.

c) Immunological tests, e.g., by agglutination tests, staining reactions with labeled antibody or precipitin testing with extracted antigen.

Some of the physiological tests, for example, the difference in sensitivity to bacitracin between Lancefield Group A and other groups of beta hemolytic streptococci (Maxted 1953), or the different bacteriophage susceptibilities among strains of Group A or Group D streptococci, are clearly relateable to cell surface biosynthesis or structure. Probably most of the immunological tests, to detect the so-called group and type antigens, are based on specific chemical structures present on or in the outermost surface layers. Thus the traditional approach to the classification of streptococci has depended heavily on features of cell surface composition. Cummins and Harris (1956) first suggested that the chemical composition of the cell wall had value as a taxonomic character. In the present article it is necessary that I proscribe mention of many interesting facets of the streptococci and limit myself primarily to those cell surface structures whose chemical nature has been investigated extensively.

From the detailed studies of Cummins and others it appeared that each bacterial genus had a characteristic pattern of amino acids while variations in the relative proportion of sugar components appeared to differentiate the species. In hydrolysates of cell wall preparations from hemolytic streptococci the amino acids, alanine, lysine and glutamic acid predominated, and the amino sugars, glucosamine and muramic acid were invariably present. Streptococcal strains of Groups A through $E$ had varying patterns of sugars present. Rhamnose was invariably present in greatest quantity and this appeared to be characteristic of streptococci. The success of this approach was exemplified by calling into question the classification of strains of Corynebacterium pyogenes, which had rhamnose, not arabinose, as a distinguishing sugar, and lysine, not diamopimelic acid, among the wall amino acids (Cummins and Harris 1956). Further investigations by other workers, however, showed that the sugar patterns of the cell walls were not always consistent among the currently recognized species. In my laboratory a nonhemolytic strain of Group A streptococci was found whose wall contained major amounts of glucose (Michel and Gooder, unpublished observations). Roberts and Stewart (1961) described a group A strain containing glucose and galactose, and Slade and Slamp (1962) described strains of Groups K, O and R which contained no rhamnose in the wall. More recently Colman and Williams (1965) compared the cell wall composition of 197 strains of streptococci. The sugar and sugar-like substances present in the wall could be arranged in 26 different patterns representing presumably clusters of strains. Very few of the currently accepted species or taxa were composed of strains showing one cell wall pattern.

It had been clearly demonstrated some years before that the group polysaccharide hapten in most streptococcal groups is located in the cell wall. While specific grouping reactions are obtained with extracted antigen and absorbed sera, this is not the situation when whole cell walls are used as antigen in agglutination tests. Multiple antigenic specificities are present which are exposed when the cells are broken to prepare walls. 
Similarly cross reactions directed against antisera of other groups are revealed. Each wall preparation reacts well with its homologous antisera but there are frequently cross-reactions at a considerably lower titer (Cummins 1962). It is possible that these overlapping antigenic specificities and sugar patterns are related to other carbohydrate wall antigens.

From these selected samples and others, I consider any attempt to classify streptococci on the basis of a qualitative pattern of sugars present in the cell wall is an unrewarding experience. At best it adds another dimension to the current classification scheme. The one possible exception is Streptococcus (Diplococcus) pneumoniae, which has a pattern containing choline, glycerol and galactosamine, a combination which I have not seen reported in any other streptococcus. A similar conclusion is drawn if instead one considers only the distribution of amino acids and amino sugars (Slade and Slamp 1962). Note for example the difficulty of separating Groups $G$ and $L$ on this basis. Other pairs would be equally difficult.

Possibly a more rational approach was to compare the chemical composition and ultimately the structure of the extracted polymers, both mucopeptide and carbohydrate haptens, which occur in the streptococcal cell wall. The wall carbohydrate haptens form the basis of the Lancefield grouping method except in Groups D and N. The elegant work of McCarty and Krause, summarized in Krause (1963), demonstrated that in the Group A streptococcus the antigenic determinants are terminal $\mathrm{N}$-acetylglucosaminide residues linked in a Beta configuration to a rhamnose-oligosaccharide which forms the backbone of the carbohydrate. Strains of streptococci have been isolated which quantitatively contain less of the terminal $\mathrm{N}$-acetylglucosamine (A-intermediate) or practically none (A-variant). Similar studies on the cell wall hapten of Group C streptococci revealed a similar situation but in this group the antigenic determinant is a terminal $\mathrm{N}$-acetyl galactosamine residue linked to the rhamnose oligosaccharide. In a manner similar to Group A streptococci, Group $C$ strains have been isolated with a quantitative variation in the amount of terminal Beta galactosaminide ( $C$ intermediate, $C$ variant).

Further studies by Krause and co-workers (Curtis and Krause 1964a, $1964 \mathrm{~b}, 1964 \mathrm{c}$ ) showed that in Groups B and G streptococci rhamnose is the determinant of the antigenic specificity of the group carbohydrate. Thus the group specificity of the immunological reaction is probably dependent on either the linkage of the terminal rhamnose to the rest of the molecule or the particular sugar which occupies the first subterminal position. Utilizing a similar approach, Michel and Willers (1964) demonstrated that the antigenic specificity of the Group $F$ carbohydrate probably resides in a terminally linked $\beta-D$-glucopyranosyl-N-acetyl-Dgalactosamine. More recently in similar studies, Willers (1966) and Willers and Michel (1966) have reported on the type antigens of Groups $F$ and D. It would appear from the reports mentioned and other studies that the basis for the serological classification of streptococci into groups and some groups into types on the basis of cell wall antigens lies in a specific chemical configuration of terminal and/or subterminal monosaccharides or amino sugars located in the streptococcal wall.

Streptococcal walls contain, in addition to the polysaccharide macromblecule, the glycosaminopeptide or mucopeptide structure. Initially it was hoped that this would be a convenient marker to distinguish between 
clusters of streptococci. Initial studies were made with whole wall preparations but qualitatively all streptococcal walls had a preponderance of alanine, lysine and glutamic acid among the wall amino acids (Cummins and Harris 1956). In Group A streptococci the amino acids ratio for the principally occurring amino acids was constant for a wide variety of serotypes and appeared unaffected by the culture medium employed (Michel and Gooder 1962). The ratio alanine:lysine:glutamic acid was $4: 1: 1$. Similar ratios were found in the walls of streptococci of other Lancefield groups by us and by many other workers. Krause and McCarty (1961) demonstrated that the amino acids were present solely in the mucopeptide layer which remained intact after the extraction of the group polysaccharide. The chemical composition of the mucopeptide appears to remain constant and is unrelated to the complexity of the commercial medium on which the organisms are grown. In synthetic medium under special conditions exceptions are known, for example hydroxylysine which occurs in $\mathbf{S}$. faecium strain 9790 after depletion of lysine from the medium (Shockman et al. 1965). It would appear, however, that in a streptococcal group recognized immunologically by means of a wall polysaccharide there is a common mucopeptide structure. Mucopeptide structure and its use as a taxonomic character has recently been extensively discussed by Ghuysen (1968).

In the streptococcal groups where the determining characteristic for classification is not a cell wall polysaccharide it would not be unexpected to find variation in the mucopeptide composition. The prime example is the enterococci as defined by Sherman (1937). All strains possess the Group D antigen, a glycerol teichoic acid located between the wall and membrane. (For review see Archibald and Baddiley, 1966). Recent extensive investigations by Deibel and others (see Deibel 1964) have shown that the Group D streptococci can be separated by physiological tests into two groups, the enterococci and the $S$. bovis $-S$. equinus group. The enterococci are further subdivided by a number of tests into $S$. faecalis and S. faecium. Ghuysen et al. (1961) had reported the presence of

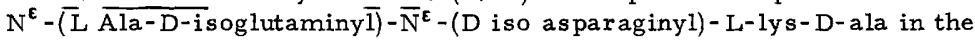
mucopeptide of a strain called S. faecalis, i. e., Lys-Asp type of mucopeptide. Similar reports were made by Ikawa (1961). However, Schleifer and Kandler (1961a, 1961b) had reported that in S. faecalis mucopeptide the interpeptide bridge consisted of a tripeptide $\mathrm{N}^{\varepsilon}-$ (L Ala $-\mathrm{L}$ Ala-L Ala) L-lys, i. e., Lys-(Ala) ${ }_{3}$ type of bridge. Thus it appeared that two different types of mucopeptide structure appeared in $\mathbf{S}$. faecalis strains. However, Kandler et al. (1968) compared 75 strains of enterococci received under a variety of names: $\underline{S}$. faecalis, including varieties liquefaciens and zymogenes; $\mathrm{S}$. faecium, including varieties durans and mobilis. Two clear-cut patterns of mucopeptide composition emerged. These patterns contained the Lys-(Ala) ${ }_{3}$ interpeptide bridge or the Lys-Asp bridge. The Lys-(Ala) ${ }_{3}$ type of structure was as sociated with strains previously classified as S. faecalis and the Lys-Asp bridge with S. faecium. Varieties of $\mathrm{S}$. faecalis and $\mathrm{S}$. faecium can be recognized by physiologic tests but I think the distinction between the major groups or species is clear and this example demonstrates the usefulness of surveying a large number of strains which are usually considered together because of the possession of a single characteristic which is not cell wall-associated.

Table 1 is an attempt to summarize the present knowledge regarding the reported structures of the interpeptide bridges discovered in groups 
TABLE 1. PEPTIDOGLYCANS IN STREPTOCOCCI ${ }^{a)}$

Peptive subunit $=\mathbb{N}^{\alpha}(L-A 1 a-D-i$ so $\left.G i n)-L-L y s-D-A\right] a$

Genus and species

S. pneumoniae

S. pyogenes

$($ Group $\bar{A})$

Enterococci

S. faecal is

S. faecium

(and varieties)

Viridans

S. thermophilus

Lactic group

(Group iv)
Interpeptide bridge ${ }^{\text {b) }}$

$$
\left.N^{\varepsilon}-(\quad \text { ? ? ? }) \text {-L-Lys }{ }^{c}\right)
$$$$
N^{\varepsilon}-(L-A 1 a-L-A 1 a)-L-L y s
$$

$N^{\varepsilon}-(L-A 1 a-L-A 1 a+L-A 7 a)-L-L y s$

$N^{\varepsilon}-(D-i s o A s p N)-L-L y s$

$N^{\varepsilon}-(L-A 1 a-L-A 1 a-L-A 1 a)-L-L y s$

$M^{\varepsilon}-(D-i$ so Aspi $)-L-L y s$

(S. lactis; S. cremoris)

S. cremoris $316 \mathrm{a} ; 331 \mathrm{a}$

a) Primarily data of Munoz et a1. 1966 and Kandler et al. 1968.

b) As defined by Ghuysen, 1968 .

c) See text.

or species of streptococci. It should be emphasized that these structures have not been confirmed in all cases by enzymic degradation but are reasonably hypothesized from the quantitative composition. Five patterns have been described. It is unlikely that Streptococcus (Diplococcus) pneumoniae has an interpeptide bridge unless its composition is the same as the subunit. It is likely that the majority of streptococci recognized in the Lancefield grouping system with the exception of Group $D$ and possibly $\mathrm{N}$ have a Lys-(Ala) ${ }_{2}$ bridge. $\mathrm{S}$. faecalis and some viridians streptococci analogous to $\mathrm{S}$. themophilus have the Lys-(Ala) ${ }_{3}$ bridge, while S. faecium and many lactic streptococci have the Lys-Asp bridge. The fifth pattern Lysine-Alanine-Threonine bridge has been reported in two strains of $\mathrm{S}$. cremoris.

It appears therefore that while the qualitative and quantitative composition of the cell wall in respect to the sugars, amino sugars and amino acids has little practical use in the classification of streptococci, the specific arrangement of these substances as found in wall polymers is extremely useful. In practice this has allowed the development of simple and rapid techniques based on the immunological specificity of some of these polymers. Undoubtedly a classification scheme is possible dividing strains into groups with a common mucopeptide structure, with sub- 
groups based on the polysaccharide structure, and even further subdivision on the basis of other wall-associated polymers such as the M protein. The present terms "Lancefield group" as in Group A, B, etc., and "type" as in Group D or F, would then disappear, but one would define each group of strains by a formula related to the specific chemical structure of the polymers and the manner in which they are linked. One recent illustration is the postulated arrangement of the polymers in the wall of S. pyogenes (Muñoz et al. 1967). When the arrangement and chemical structure of the polymers in many other strains of streptococci are known it may be possible to indicate these by a series of letters or numbers for each strain.

As it is likely that all wall polymers are synthesized and integrated by a series of interrelated biochemical pathways and control mechanisms, the basis for the classification would likely be the structural end product of, say, 10-50 enzymes. Such a system has advantages over classification schemes based on single phenotypic markers and in practice is simpler to apply than the nucleic acid hybridization techniques. Whether or not this system would bear any relationship to a phylogenetic system and whether any of the groups of strains so formed will be truly "bacterial species" is of course a matter of opinion.

\section{ACKNOW LEDGMENT}

Financial support to the author is provided by Grant No. AI-04577 from the National Institutes of Health.

\section{BIBLIOGRAPHY}

Archibald, A.R. and J. Baddiley. 1966. The teichoic acids. Adv. Carbohyd. Chem. 21:323-375.

Colman, G. 1968. The application of computers to the classification of streptococci. J. Gen. Microbiol. 50:149-158.

and R.E.O. Williams. 1965. The cell walls of streptococci. J. Gen. Microbiol. 41:375-387.

Cummins, C.S. $196 \overline{2}$. Immunochemical specificity and the location of antigens in the bacterial cell. In: Microbial Classification, eds. G. C. Ainsworth and P.H.A. Sneath. Symp. Soc. Gen. Microbiol. 12:212-241.

and H. Harris. 1956. The chemical composition of the cell-wall

in some Gram-positive bacteria and its possible value as a taxonomic character. J. Gen. Microbiol. 14:583.

Curtis, S.N. and R. M. Krause. 19 $\overline{64}$ a. Immunochemical studies on the specific carbohydrate of Group G carbohydrate. J. Exp. Med. I19: 997.

and 1964b. Antigenic relationships between Groups B and

G streptococci. J. Exp. Med. 120:629.

and 1964c. The identification of rhamnose as an antigenic

determinant of Group B streptococci carbohydrates. Fed. Proc. 23: 191.

Deibel, R.H. 1964. The Group D streptococci. Bacteriol, Rev. 28:330366.

Drucker, D. B. and T.H. Melville. 1969. Computer classification of streptococci, mostly of oral origin. Nature 221:664. 
Ghuysen, J. M. 1968. Use of bacteriolytic enzymes in determination of wall structure and their role in cell metabolism. Bacteriol. Rev. 32: $425-464$

, E. Bricas, M. Leyh-Bouille, M. Lache and G. D. Shockman.

1961. The peptide $\mathrm{N}^{\varepsilon}-\left(\mathrm{L}-\mathrm{Alany} \mathrm{l}-\mathrm{D}\right.$-isoglutaminyl) $-\mathrm{N}^{\varepsilon}-(\mathrm{D}$-isoasparaginyl)-L-lysyl-D-alanine and the disaccharide $\mathrm{N}$ acetylglucosaminyl$\beta-1,4-\mathrm{N}$-acetyl-muramic acid in cell wall peptidoglycan of Streptococcus faecalis strain ATCC 9790. Biochemistry 6:2607-2619.

Ikawa, M. I96l. The partial chemical degradation of the cell walls of Lactobacillus plantarum, $\mathrm{S}$. faecalis and Lactobacillus casei. J. Biol. Chem. 236:1087-1092.

Kandler, O., K.H. Schleifer and R. Dandl. 1968. Differentiation of Streptococcus faecalis Andrewes and Harder and Streptococcus faecium Orla-Jensen based on the amino acid composition of their murein. J. Bact. 96:1935-1939.

Krause, R. M. 1963. Symposium on relationship of structure of microorganisms to their immunological properties. IV. Antigenic and biochemical composition of hemolytic streptococcal cell walls. Bacteriol. Rev. 27:369-380.

and M. McCarty. 1961. Studies on the chemical structure of the streptococcal cell wall. I. The identification of a mucopeptide in the cell walls of group A and A-variant streptococci. J. Exp. Med. 114: 127.

Mandel, M. 1969. New approaches to bacterial taxonomy: Perspective and prospects. Ann. Rev. Microbiol. 23:239-274.

Maxted, W.R. 1953. Use of bacitracin fo $\bar{r}$ identifying Group A haemolytic streptococci. J. Clin. Path. 6:224-226.

Michel, M. F. and H. Gooder. 1962. Ämino acids, amino sugars and sugars present in the cell wall of some strains of Streptococcus pyogenes. J. Gen. Microbiol. 29:199.

and J.M.N. Willers. 1964. Immunochemistry of Group F streptococci; isolation of group specific oligosaccharides. J. Gen. Microbiol. $37: 381-389$.

Muñoz, E., J. M. Ghuysen, M. Leyh-Bouille, J.F. Petit, H. Heyman, E. Bricas, and P. Lefrancier. 1966. The peptide subunit $\mathrm{N}^{\mathrm{a}}-$ (Lalanyl-D-isoglutaminyl)-L-lysyl-D-alanine in cell wall peptidoglycans of Staphylococcus aureus, strain Copenhagen, Micrococcus aureus

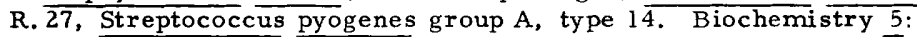
$3748-3764$.

, and H. Heymann. 1967. Cell walls of Streptococcus pyogenes type 14, C polysaccharide-peptidoglycan and $G$ polysaccharidepeptidoglycan complexes. Biochemistry 6:3659-3670.

Raj, H. and R.R. Colwell. 1966. Taxonomy of enterococci by computer analysis. Can. J. Microbiol. 12:353.

Roberts, W.S. L. and F.S. Stewart. 1961. The sugar composition of streptococcal cell walls and its relation to haemagglutination pattern. J. Gen. Microbiol. 24:253.

Schleifer, K.H. and O. Kandler, 1961a. Zur chemischen Zussammensetzung der Zellwand der Streptokokken. 1. Die aminosäuresequenz des mureins von $\underline{S}$. thermophilus und $\underline{S}$. faecalis. Arch. Microbiol. $57: 335-364$. and $\quad 1961 \mathrm{~b}$. Zur chemischen Zussammensetzung der Zellwand der Streptokokken. II. Der aminosäuresequenz des mureins von S. lactis und cremoris. Arch. Microbiol. 57:365-381. 
Sherman, J. M. 1937. The streptococci. Bacteriol. Rev. 1:3-97.

Shockman, G.D., J.S. Thompson and M. J. Conover. 1965. Replacement of lysine by hydroxylysine and its effects on cell lysis in Streptococcus faecalis. J. Bacteriol. 90:575-588.

Slade, H.D. and W.C. Slamp. 1962. Cell-wall composition and the grouping antigens of streptococci. J. Bact. 84:345-351.

Weismann, S. M., P.R. Reich, N. L. Somerson and R. M. Cole. 1966. Genetic differentiation by nucleic acid homology. IV. Relationships among Lancefield groups and serotypes of streptococci. J. Bact. 92 : 1372-1377.

Willers, J. M. N. 1966. Immunochemistry of Group F streptococci: Isolation of the determinant group of a type III variant strain. Antonie von Leeuwenhoek 32:458.

and M.F. Michel. 1966. Immunochemistry of the type antigen of Streptococcus faecalis. Antonie von Leeuwenhoek 32:337-338. 